International Journal of Pure and Applied Mathematics

Volume 106 No. 2 2016, 523-531

ISSN: 1311-8080 (printed version); ISSN: 1314-3395 (on-line version)

url: http://www.ijpam.eu

doi: 10.12732/ijpam.v106i2.15

\title{
HAUSDROFF PROPERTY OF CARTESIAN AND TENSOR PRODUCT OF GRAPHS
}

\author{
Seena $\mathrm{V}^{1} \S$, Raji Pilakkat ${ }^{2}$ \\ ${ }^{1,2}$ Department of Mathematics \\ University of Calicut \\ Calicut University \\ Malappuram (District), PIN 673 635, Kerala, INDIA \\ rajiunical@rediffmail.com
}

Abstract: A simple graph $G$ is said to be Hausdroff if for any two distinct vertices $u$ and $v$ of $G$, one of the following conditions hold:

1. Both $u$ and $v$ are isolated

2. Either $u$ or $v$ is isolated

3. There exist two nonadjacent edges $e_{1}$ and $e_{2}$ of $G$ such that $e_{1}$ is incident with $u$ and $e_{2}$ is incident with $v$.

In this paper we derive sufficient conditions for cartesian and tensor products of two graphs to be Hausdroff.

AMS Subject Classification: $05 \mathrm{C} 76$

Key Words: Hausdroff graph, isolated vertex, Cartesian product, end-block, tensor product

\section{Introduction}

All the graphs considered here are finite and simple. In this paper we denote the set of vertices of $G$ by $V(G)$, the set of edges of $G$ by $E(G)$ and the minimum degree of $G$ by $\delta(G)$.

Received: September 25, 2015

Published: February 15, 2016

$\S_{\text {Correspondence author }}$ (c) 2016 Academic Publications, Ltd.

url: www.acadpubl.eu 
The degree [4] of a vertex $v$ in a graph $G$, denoted by $\operatorname{deg} v$, is the number of edges incident with $v$. A pendant vertex [6] in a graph $G$ is a vertex of degree one. A vertex $v$ is isolated [2] if $\operatorname{deg} v=0$. By an empty graph [5] we mean a graph with no edges. Two vertices $u$ and $v$ of $G$ are adjacent [8], if $u v$ is an edge of $G$. A simple graph is said to be complete[7] if every pair of distinct vertices of $G$ are adjacent in $G$. A complete graph of $n$ vertices is denoted by $K_{n}$. A connected graph that has no cut vertices is called a block [5]. A block of $G$ containing exactly one cut vertex of $G$ is called an end-block [3] of $G$. The Cartesian product [9] $G \square H$ of two graphs $G=(V(G), E(G))$ and $H=(V(H), E(H))$ is the graph with vertex set $V(G) \times V(H)$ where the vertex $\left(u_{1}, v_{1}\right)$ is adjacent to the vertex $\left(u_{2}, v_{2}\right)$ whenever $u_{1} u_{2} \in E(G)$ and $v_{1}=v_{2}$, or $u_{1}=u_{2}$ and $v_{1} v_{2} \in E(H)$. The Tensor product(or direct product)[1] $G \times H$ of two graphs $G$ and $H$ is the graph with the vertex set $V(G) \times V(H)$, two vertices $\left(u_{1}, v_{1}\right)$ and $\left(u_{2}, v_{2}\right)$ being adjacent in $G \times H$ if, and only if, $u_{1} u_{2} \in E(G)$ and $v_{1} v_{2} \in E(H)$. A graph $G$ is said to be Hausdroff [10] if for any two distinct vertices $u$ and $v$ of $G$, one of the following three conditions hold: (1) Both $u$ and $v$ are isolated (2) Either $u$ or $v$ is isolated (3) There exist two nonadjacent edges $e_{1}$ and $e_{2}$ of $G$ such that $e_{1}$ is incident with $u$ and $e_{2}$ is incident with $v$. From the definition of a Hausdroff graph we have if $G$ is a graph with $\delta(G)=1$, then it cannot be Hausdroff. In particular $K_{2}$ is not Hausdroff. Also if $G$ is Hausdroff, then any supergraph of $G$ is Hausdroff.

Theorem 1. [10] Let $G=(V(G), E(G))$ be a graph with $\delta(G) \geq 3$ then, $G$ is Hausdroff.

\section{Cartesian Product}

From the definition of cartesian product of graphs we have:

Proposition 2. The cartesian product $K_{n} \square K_{n}$ is Hausdroff for every $n$.

Proposition 3. The cartesian product $K_{n} \square K_{m}(n \neq m)$ is Hausdroff if, and only if, either $n=1$ and $m \geq 4$ or $n \geq 2$ and $m \geq 2$.

Corollary 4. The cartesian product $C_{n} \square C_{m}$ is Hausdroff $\forall n, m$.

Proposition 5. The cartesian product $P_{n} \square P_{m}$ is Hausdroff $\forall n, m$. Example 6.

Example 6 shows that the cartesian product of two non-Hausdroff graphs can be Hausdroff. 




Figure 1: Cartesian product of paths $P_{1}$ and $P_{2}$

Theorem 7. Let $G_{1}$ and $G_{2}$ be two graphs with no isolated vertices. Then $G_{1} \square G_{2}$ is Hausdroff.

Proof. Let $\left(u_{i}, v_{j}\right)$ and $\left(u_{r}, v_{s}\right)$ be two distinct vertices of $G_{1} \square G_{2}$.

Case 1. $u_{i}=u_{r}$

Then the vertices $v_{j}$ and $v_{s}$ of $G_{2}$ are distinct. Since $G_{1}$ is a graph with no isolated vertices, $G_{1}$ contains a vertex $u_{p}$ such that $u_{i}$ and $u_{p}$ are adjacent in $G_{1}$. Then $\left(u_{i}, v_{j}\right)\left(u_{p}, v_{j}\right)$ and $\left(u_{i}, v_{s}\right)\left(u_{p}, v_{s}\right)$ are two nonadjacent edges of $G_{1} \square G_{2}$.

Case 2. $u_{i} \neq u_{r}$

In this case, either $v_{j}=v_{s}$ or $v_{j} \neq v_{s}$. Suppose $v_{j}=v_{s}$. Since $G_{1} \square G_{2}=$ $G_{2} \square G_{1}$, the result follows as in Case 1. So we need only to consider the case $v_{j} \neq$ $v_{s}$. In this case if $u_{i}$ is adjacent to $u_{r}$, then $\left(u_{i}, v_{j}\right)\left(u_{r}, v_{j}\right)$ and $\left(u_{r}, v_{s}\right)\left(u_{i}, v_{s}\right)$ are two nonadjacent edges of $G_{1} \square G_{2}$ incident with $\left(u_{i}, v_{j}\right)$ and $\left(u_{r}, v_{s}\right)$ respectively. If $u_{i}$ is not adjacent to $u_{r}$, since $G_{1}$ is free from isolated vertices, there exist vertices $u_{p}$ and $u_{q}$ distinct from $u_{i}$ and $u_{r}$ such that $u_{i}$ is adjacent to $u_{p}$ and $u_{r}$ is adjacent to $u_{q}$ in $G_{1}$. $\left(u_{p}\right.$ may be equal to $\left.u_{q}\right)$ Then $\left(u_{i}, v_{j}\right)\left(u_{p}, v_{j}\right)$ and $\left(u_{r}, v_{s}\right)\left(u_{q}, v_{s}\right)$ are two nonadjacent edges of $G_{1} \square G_{2}$ incident with $\left(u_{i}, v_{j}\right)$ and $\left(u_{r}, v_{s}\right)$ respectively.

Hence the theorem.

Remark 8. Theorem 7 need not be true if both the graphs contain isolated vertices. (see. Figure [2]).

If $G_{1}$ contains an isolated vertex $u$ and $G_{2}$ contains a pendant edge $v w$ then $(u, v)(u, w)$ is a pendant edge of the cartesian product $G_{1} \square G_{2}$ of $G_{1}$ and $G_{2}$. Thus in such cases $G_{1} \square G_{2}$ can never be Hausdroff. We state this result as a proposition as follows: 

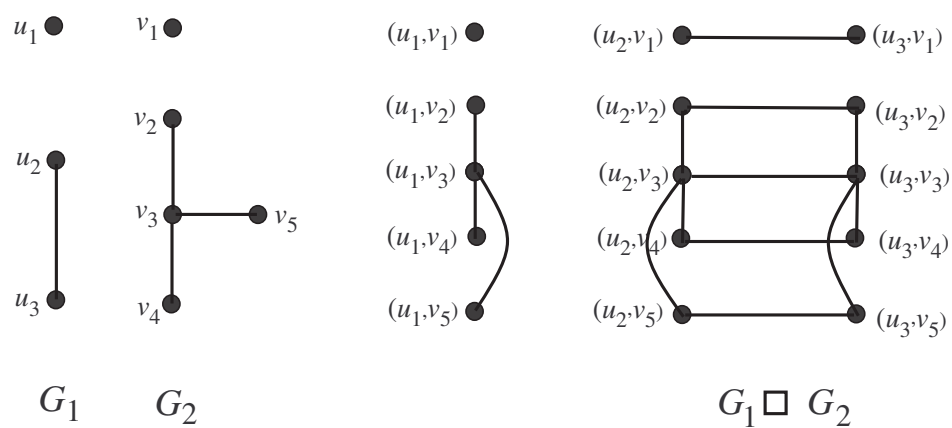

$G_{1} \square G_{2}$

Figure 2: Cartesian product of graphs


$G_{1} \square G_{2}$

Figure 3: Cartesian product of graphs

Proposition 9. The cartesian product $G_{1} \square G_{2}$ of two graphs $G_{1}$ and $G_{2}$ is not Hausdroff if $\delta\left(G_{1}\right)=0$ and $\delta\left(G_{2}\right)=1$.

The question then arise is that what happens to the cartesian product when we increase the minimum degree of the graph $G_{2}$. Unfortunately, the result remains failed in certain cases. For example, consider the graphs $G_{1}$ and $G_{2}$ and their cartesian product in Figure 3. There $\delta\left(G_{1}\right)=0$ and $\delta\left(G_{2}\right)=2$. The cartesian product $G_{1} \square G_{2}$ of $G_{1}$ and $G_{2}$ contains a triangle, hence it cannot be Hausdroff.

\section{Example 10.}

But one can overcome this difficult situation by giving some restrictions to the graph $G_{2}$.

Theorem 11. Let $G_{1}$ be any graph and $G_{2}$ be a graph with no triangle as end-block. If $\delta\left(G_{2}\right)=2$, then $G_{1} \square G_{2}$ is Hausdroff. 
Proof. Let $\left(u_{i}, v_{j}\right)$ and $\left(u_{r}, v_{s}\right)$ be two distinct vertices of $G_{1} \square G_{2}$.

Case 1. $u_{i}=u_{r}$

Suppose $v_{j}$ and $v_{s}$ are adjacent vertices of $G_{2}$. Since $\delta\left(G_{2}\right)=2$, there exists a vertex $v_{p}$ distinct from $v_{s}$ such that $v_{j}$ is adjacent to $v_{p}$. If $v_{s}$ and $v_{p}$ are not adjacent then $v_{s}$ must be adjacent to some vertex $v_{q}$ of $G_{2}$ distinct from $v_{j}$ and $v_{p}$. Then $\left(u_{i}, v_{j}\right)\left(u_{i}, v_{p}\right)$ and $\left(u_{i}, v_{s}\right)\left(u_{i}, v_{q}\right)$ are two nonadjacent edges of $G_{1} \square G_{2}$ incident with $\left(u_{i}, v_{j}\right)$ and $\left(u_{r}, v_{s}\right)$ respectively.

If $v_{s}$ and $v_{p}$ are adjacent then the subgraph induced by the vertices $v_{j}, v_{s}, v_{p}$ is a triangle in $G_{2}$. Since $G_{2}$ contains no triangle as end-block either $v_{s}$ is adjacent to a vertex $v_{q}$ distinct from $v_{j}$ and $v_{p}$ or $v_{j}$ is adjacent to a vertex $v_{t}$ distinct from $v_{s}$ and $v_{p}$. In the first case $\left(u_{i}, v_{j}\right)\left(u_{i}, v_{p}\right)$ and $\left(u_{i}, v_{s}\right)\left(u_{i}, v_{q}\right)$ are two nonadjacent edges of $G_{1} \square G_{2}$. In the second case $\left(u_{i}, v_{j}\right)\left(u_{i}, v_{t}\right)$ and $\left(u_{i}, v_{s}\right)\left(u_{i}, v_{p}\right)$ are two nonadjacent edges of $G_{1} \square G_{2}$.

Suppose $v_{j}$ and $v_{s}$ are not adjacent in $G_{2}$. In this case since $\delta\left(G_{2}\right)=2$, we can choose two distinct vertices $v_{p}$ and $v_{q}$ of $G_{2}$ such that $v_{p}$ is adjacent to $v_{j}$ and $v_{q}$ is adjacent to $v_{s}$, then $\left(u_{i}, v_{j}\right)\left(u_{i}, v_{p}\right)$ and $\left(u_{i}, v_{s}\right)\left(u_{i}, v_{q}\right)$ are two nonadjacent edges of $G_{1} \square G_{2}$ incident with $\left(u_{i}, v_{j}\right)$ and $\left(u_{r}, v_{s}\right)$ respectively.

Case 2. $u_{i} \neq u_{r}$

Suppose $v_{j}=v_{s}$, since $\delta\left(G_{2}\right)=2$, there exist two distinct vertices $v_{p}$ and $v_{q}$ such that $v_{j}$ is adjacent to both $v_{p}$ and $v_{q}$. Then $\left(u_{i}, v_{j}\right)\left(u_{i}, v_{p}\right)$ and $\left(u_{r}, v_{s}\right)\left(u_{r}, v_{q}\right)$ are two nonadjacent edges of $G_{1} \square G_{2}$.

Suppose $v_{j} \neq v_{s}$, then by proceeding as in the proof of Case 1 we get two nonadjacent edges incident with the vertices $\left(u_{i}, v_{j}\right)$ and $\left(u_{r}, v_{s}\right)$. First of all we consider the case $v_{j}$ and $v_{s}$ are adjacent vertices of $G_{2}$. Since $\delta\left(G_{2}\right)=2$, there exists a vertex $v_{p}$ distinct from $v_{s}$ such that $v_{p}$ is adjacent to $v_{j}$. If $v_{s}$ and $v_{p}$ are not adjacent then $v_{s}$ must be adjacent to some vertex $v_{q}$ of $G_{2}$ distinct from $v_{j}$ and $v_{p}$. Which implies $\left(u_{i}, v_{j}\right)\left(u_{i}, v_{p}\right)$ and $\left(u_{i}, v_{s}\right)\left(u_{i}, v_{q}\right)$ are two nonadjacent edges of $G_{1} \square G_{2}$ incident with $\left(u_{i}, v_{j}\right)$ and $\left(u_{r}, v_{s}\right)$ respectively. If $v_{s}$ and $v_{p}$ are adjacent then $v_{j}, v_{s}, v_{p}$ form a triangle. Since $G_{2}$ contains no triangle as end-block either $v_{s}$ is adjacent to a vertex $v_{q}$ distinct from $v_{j}$ and $v_{p}$ or $v_{j}$ is adjacent to a vertex $v_{t}$ distinct from $v_{s}$ and $v_{p}$. In the first case $\left(u_{i}, v_{j}\right)\left(u_{i}, v_{p}\right)$ and $\left(u_{r}, v_{s}\right)\left(u_{r}, v_{q}\right)$ are two nonadjacent edges of $G_{1} \square G_{2}$. In the second case $\left(u_{i}, v_{j}\right)\left(u_{i}, v_{t}\right)$ and $\left(u_{r}, v_{s}\right)\left(u_{r}, v_{p}\right)$ are two nonadjacent edges of $G_{1} \square G_{2}$.

Suppose $v_{j}$ and $v_{s}$ are not adjacent in $G_{2}$. In this case, since $\delta\left(G_{2}\right)=2$, we can choose two distinct vertices $v_{p}$ and $v_{q}$ of $G_{2}$ such that $v_{j}$ is adjacent to $v_{p}$ and $v_{s}$ is adjacent to $v_{q}$. Then, $\left(u_{i}, v_{j}\right)\left(u_{i}, v_{p}\right)$ and $\left(u_{r}, v_{s}\right)\left(u_{r}, v_{q}\right)$ are two nonadjacent edges of $G_{1} \square G_{2}$.

Thus in all the cases we have proved that for any two distinct vertices 
$\left(u_{i}, v_{j}\right),\left(u_{r}, v_{s}\right)$ of $G_{1} \square G_{2}$ there exist two nonadjacent edges $e_{1}$ and $e_{2}$ of $G_{1} \square G_{2}$ such that $e_{1}$ is incident with $\left(u_{i}, v_{j}\right)$ and $e_{2}$ is incident with $\left(u_{r}, v_{s}\right)$. Hence $G_{1} \square G_{2}$ is Hausdroff.

Theorem 12. Let $G_{1}$ be any graph and $G_{2}$ be a graph with $\delta\left(G_{2}\right) \geq 3$, then $G_{1} \square G_{2}$ is Hausdroff.

Proof. Let $(u, v) \in V\left(G_{1} \square G_{2}\right)$, then $u \in V\left(G_{1}\right)$ and $v \in V\left(G_{2}\right)$. Since $\delta\left(G_{2}\right) \geq 3, v$ is adjacent to at least three vertices, say $v_{1}, v_{2}, v_{3}$ of $G_{2}$. Then the vertex $(u, v)$ is adjacent to the vertices $\left(u, v_{1}\right),\left(u, v_{2}\right)$ and $\left(u, v_{3}\right)$ of $G_{1} \square G_{2}$. Therefore, $\operatorname{deg}(u, v) \geq 3$. Since $(u, v)$ is an arbitrary vertex of $G_{1} \square G_{2}, \delta\left(G_{1} \square G_{2}\right)$ $\geq 3$. Hence by Theorem $1, G_{1} \square G_{2}$ is Hausdroff.

Proposition 13. Let $G_{1}$ be an empty graph and $G_{2}$ be a Hausdroff graph then $G_{1} \square G_{2}$ is Hausdroff.

Proof. Let $\left\{u_{1}, u_{2}, \ldots, u_{n}\right\}$ and $\left\{v_{1}, v_{2}, \ldots, v_{m}\right\}$ be the vertex sets of $G_{1}$ and $G_{2}$ respectively. For $i=1,2, \ldots, n$, let $H_{i}=\left\langle\left\{\left(u_{i}, v_{j}\right) ; j=1,2, \ldots, m\right\}\right\rangle$. Then $G_{1} \square G_{2}=\bigcup_{i=1}^{n} H_{i}$. Note that for every $i, H_{i}$ is isomorphic to $G_{2}$ and hence Hausdroff. Therefore, the graph $G_{1} \square G_{2}$, being the union of Hausdroff graphs, is Hausdroff.

Remark 14. Since $G_{1} \square G_{2}=G_{2} \square G_{1}$, Propositions 9, 13 and Theorems $7,11,12$ are still true even if we interchange the roles of $G_{1}$ and $G_{2}$.

Theorem 15. Cartesian product of two Hausdroff graphs is Hausdroff.

Proof. Let $G_{1}$ and $G_{2}$ be two Hausdroff graphs. Then, for $i=1,2, G_{i}=$ $K_{i} \cup H_{i}$, where $V\left(K_{i}\right)$ is the set all isolated vertices of $G_{i}$ and $V\left(H_{i}\right)$ is the set of all non-isolated vertices of $G_{i}$. Hence $G_{1} \square G_{2}=\left(K_{1} \square K_{2}\right) \cup\left(K_{1} \square H_{2}\right) \cup$ $\left(H_{1} \square K_{2}\right) \cup\left(H_{1} \square H_{2}\right)$. By Proposition 13, $\left(K_{1} \square K_{2}\right),\left(K_{1} \square H_{2}\right)$ and $\left(H_{1} \square K_{2}\right)$ are Hausdroff. By Theorem 7, $H_{1} \square H_{2}$ is Hausdroff. Therefore, $G_{1} \square G_{2}$ is Hausdroff. 


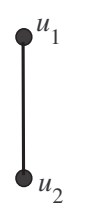

$K_{2}$



$K_{3}$

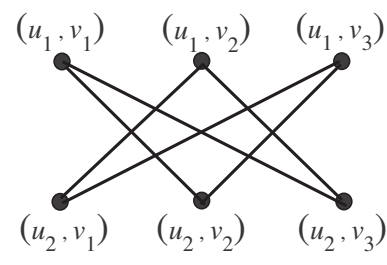

$K_{2} \times K_{3}$

Figure 4: Tensor product of $K_{2}$ and $K_{3}$

\section{Tensor Product}

Another interesting graph product that we can consider is that of the tensor product. Let us start with the tensor product of $K_{2}$ and $K_{3}$. Though both $K_{2}$ and $K_{3}$ are non-Hausdroff their tensor product seems to be Hausdroff.

Note that the graph $K_{2}$ is free from isolated vertices and the graph $K_{3}$ has minimum degree 2. Lemma 16 shows that this result is true in general. That is, if $\delta\left(G_{1}\right) \geq 1$ and $\delta\left(G_{2}\right)=0$, then $G_{1} \times G_{2}$ is Hausdroff.

Lemma 16. Let $G_{1}$ be a graph with no isolated vertices and $G_{2}$ be a graph with $\delta\left(G_{2}\right)=2$. Then $G_{1} \times G_{2}$ is Hausdroff.

Proof. Let $V\left(G_{1}\right)=\left\{u_{i}, i=1,2, \ldots m\right\}$ and $V\left(G_{2}\right)=\left\{v_{j}, j=1,2, \ldots n\right\}$. Then, $V\left(G_{1} \times G_{2}\right)=\left\{\left(u_{i}, v_{j}\right) ; i=1,2, \ldots m, j=1,2, \ldots n\right\}$. Consider two distinct vertices $\left(u_{i}, v_{j}\right)$ and $\left(u_{r}, v_{s}\right)$ of $G_{1} \times G_{2}$. Since $G_{1}$ contains no isolated vertices, the vertex $u_{i}$ is adjacent to at least one vertex of $G_{1}$.

Case 1. $u_{i}$ and $u_{r}$ are adjacent vertices of $G_{1}$.

Since $\delta\left(G_{2}\right)=2$, there exists a vertex $v_{p}$ distinct from $v_{s}$ such that $v_{p}$ is adjacent to $v_{j}$. Similarly there exists a vertex $v_{q}$ distinct from $v_{j}$ such that $v_{q}$ is adjacent to $v_{s}$. Then $\left(u_{i}, v_{j}\right)\left(u_{r}, v_{p}\right)$ and $\left(u_{r}, v_{s}\right)\left(u_{i}, v_{q}\right)$ are two nonadjacent edges of $G_{1} \times G_{2}$ incident with $\left(u_{i}, v_{j}\right)$ and $\left(u_{r}, v_{s}\right)$ respectively.

Case 2. $u_{i}$ and $u_{r}$ are nonadjacent vertices of $G_{1}$.

Choose vertices $u_{p}$ and $u_{q}$ of $G_{1}$ which are adjacent to the vertices $u_{i}$ and $u_{r}$ respectively. If $v_{j}$ and $v_{s}$ are adjacent in $G_{2}$ then, the edges $\left(u_{i}, v_{j}\right)\left(u_{p}, v_{s}\right)$ and $\left(u_{r}, v_{s}\right)\left(u_{q}, v_{j}\right)$ are two nonadjacent edges of $G_{1} \times G_{2}$ incident with $\left(u_{i}, v_{j}\right)$ and $\left(u_{r}, v_{s}\right)$ respectively. Otherwise, since $\delta\left(G_{2}\right)=2$, we can choose two distinct vertices $v_{p}$ and $v_{q}$ of $G_{2}$ such that $v_{p}$ is adjacent to $v_{j}$ and $v_{q}$ is adjacent to $v_{s}$. Then, the edges $\left(u_{i}, v_{j}\right)\left(u_{p}, v_{p}\right)$ and $\left(u_{r}, v_{s}\right)\left(u_{q}, v_{q}\right)$ are two nonadjacent edges of $G_{1} \times G_{2}$ incident with $\left(u_{i}, v_{j}\right)$ and $\left(u_{r}, v_{s}\right)$ respectively. 
Theorem 17 shows that the restriction $\delta\left(G_{2}\right)=2$ on the second graph $G_{2}$ can be withdrawn.

Theorem 17. Let $G_{1}$ be a graph with no isolated vertices and $G_{2}$ be a graph with $\delta\left(G_{2}\right) \geq 2$. Then, the tensor product $G_{1} \times G_{2}$ of $G_{1}$ and $G_{2}$ is Hausdroff.

Proof. If $\delta\left(G_{2}\right)=2$, then the proof follows from Lemma 16. Now suppose $\delta\left(G_{2}\right) \geq 3$. Let $(u, v)$ be a vertex of $G_{1} \times G_{2}$. Since $G_{1}$ is a graph with no isolated vertices, the vertex $u$ is adjacent to at least one vertex say $w$ of $G_{1}$. Since $\delta\left(G_{2}\right) \geq 3$, the vertex $v$ is adjacent to at least three vertices say $v_{1}, v_{2}, v_{3}$ of $G_{2}$. Then the vertex $(u, v)$ is adjacent to the vertices $\left(w, v_{1}\right),\left(w, v_{2}\right)$, and $\left(w, v_{3}\right)$ of $G_{1} \times G_{2}$. Therefore, $\operatorname{deg}(u, v) \geq 3$. Since $(u, v)$ is arbitrary, $\delta\left(G_{1} \times G_{2}\right) \geq 3$. Hence by Theorem $1, G_{1} \times G_{2}$ is Hausdroff.

Lemma 18. If one of $G_{1}$ and $G_{2}$ be empty graphs then, $G_{1} \times G_{2}$ is Hausdroff.

Proof. Let one of $G_{1}$ and $G_{2}$ be empty graphs then, $G_{1} \times G_{2}$ is an empty graph. This implies $G_{1} \times G_{2}$ is Hausdroff.

Theorem 19. Let $G_{1}$ be any graph and $G_{2}$ be a graph with $\delta\left(G_{2}\right) \geq 2$, then $G_{1} \times G_{2}$ is Hausdroff.

Proof. We can write $G_{1}=K \cup H$, where $V(K)$ is the set all isolated vertices of $G_{1}$ and $V(H)$ is the set all non-isolated vertices of $G_{1}$. Then $G_{1} \times G_{2}=$ $\left(K \times G_{2}\right) \cup\left(H \times G_{2}\right)$. Since $K$ is empty by Lemma $18, K \times G_{1}$ is Hausdroff. By Theorem 17, $H \times G_{2}$ is Hausdroff. Therefore, the graph $G_{1} \times G_{2}$, being the union of Hausdroff graphs, is Hausdroff.

Corollary 20. For every $n, m \geq 3, C_{n} \times C_{m}$ is Hausdroff.

Theorem 21. Let $G_{1}$ and $G_{2}$ be two graphs such that both $G_{1}$ and $G_{2}$ contain at least one pendant vertex. Then $G_{1} \times G_{2}$ can never be Hausdroff.

Proof. Let $u$ be a pendant vertex with pendant edge $u x$ in $G_{1}$ and let $v$ be a pendant vertex with $u y$ as pendant edge in $G_{2}$. Then $(u, v)(x, y)$ is a pendant edge in $G_{1} \times G_{2}$. Therefore, $G_{1} \times G_{2}$ is not Hausdroff.

Theorem 22. Tensor product of any two Hausdroff graphs is Hausdroff. 
Proof. Let $G_{1}$ and $G_{2}$ be the given Hausdroff graphs. For $i=1,2$, we write $G_{i}=K_{i} \cup H_{i}$, where $V\left(K_{i}\right)$ is the set all isolated vertices of $G_{i}$ and $V\left(H_{i}\right)$ is the set all non-isolated vertices of $G_{i}$. Then $G_{1} \times G_{2}=\left(K_{1} \times K_{2}\right) \cup\left(K_{1} \times H_{2}\right) \cup$ $\left(H_{1} \times K_{2}\right) \cup\left(H_{1} \times H_{2}\right)$. Since both $K_{1}$ and $K_{2}$ are empty graphs by Lemma 18, $K_{1} \times K_{2}, K_{1} \times H_{2}, H_{1} \times K_{2}$ are Hausdroff. By Theorem $19, H_{1} \times H_{2}$ is Hausdroff. Therefore, the graph $G_{1} \times G_{2}$, being the finite union of Hausdroff graphs, is Hausdroff.

\section{Conclusion}

In this paper we have discussed conditions under which Cartesian product of two graphs is Hausdroff. It is identified that Cartesian product of two Hausdroff Graphs is Hausdroff. Conditions under which Tensor product of two graphs become Hausdroff have been formulated. There are many unsolved problems in this area which are yet to be settled.

\section{Acknowledgments}

The first author acknowledge the financial support by University Grants Commission of India, under Faculty Development Programme and from CSIR.

\section{References}

[1] Bostjan Bresar, Simon Spacapan, On the connectivity of the direct product of graphs, Australasian journal of combinatorics, 41 (2008), 45-56.

[2] Douglas B. West, Introduction to Graph Theory, Prentice-Hall, USA (2000).

[3] Gary Chartrand, Ping Zhang, Chromatic Graph Theory, CRC Press, USA (2009).

[4] F. Harary, Graph Theory, Narosa Publishing House, India (1990).

[5] J. A. Bondy, U.S.R. Murty, Graph Theory, Springer Verlag, USA (2008).

[6] K.R Parthasarathy, Basic Graph Theory, McGraw-Hill Professional Publishing, USA(1994).

[7] R. Balakrishnan, K. Ranganathan, A Text Book of Graph Theory, Springer Verlag, USA (2000).

[8] Reinhard Diestel, Graph Theory, Springer Verlag, USA (1997).

[9] Sandi Klavzar, Alenka Lipovec, Marko Petkovsek 2, On subgraphs of Cartesian product graphs, Discrete Mathematics, 244 (2002), 223-230.

[10] V. Seena, Raji Pilakkat, Hausdroff Graphs, British Journal of Mathematics and Computer Science, 12(1), (2016), 1-12. 
